\title{
Application of different types of forward modeling results in seismic reservoir prediction
}

\author{
Jinlong $L v^{1, a}$ \\ ${ }^{1}$ Exploration and Development Research Institute of Daqing Oilfield Co. Ltd. Dqing,China \\ avjinlong@petrochina.com.cn
}

Keywords: reservoir prediction, forward modeling, transitional rocks, seismic attribute extraction. Abstract. There are a large number of transitional rocks (silty mudstone and muddy silty sand) in the Fuyu reservoir, which affect the seismic characteristics and reduce the reservoir prediction accuracy. The lithology P-wave impedance log can be created by the well log interpretation result. The lithology $\mathrm{P}$-wave impedance log, real $\mathrm{P}$-wave impedance log and space variant wavelet are used to calculate two forward modelings, and the difference between the two results can shield the effect of the transitional rocks, obtain a reliable seismic data. The seismic attributes are extracted by using the partition window, and the accuracy of reservoir prediction is improved from $53.9 \%$ to $75.1 \%$. The coincidence rate of well is greatly improved, and the method can get good reservoir prediction results.

\section{Background}

The research area is located in the eastern part of Songliao Basin, and the whole trend is from southeast to northwest, while the southeast is slow and the northwest is steep. The fluvial facies dominated sedimentary system of Fuyu oil layer is mainly controlled by the source of the southwest. Reservoir belongs to low porosity, low permeability channel sand body formation on the upper and lower reservoir lithologic reservoir ${ }^{[1]}$. Through seismic data and cored well data analysis, it is found that the transition lithologies (powder sandy mudstone, mud silty sandstone) reservoir prediction has great influence on. Therefore, we can use the different types of forward simulation to carry out seismic reservoir prediction research.

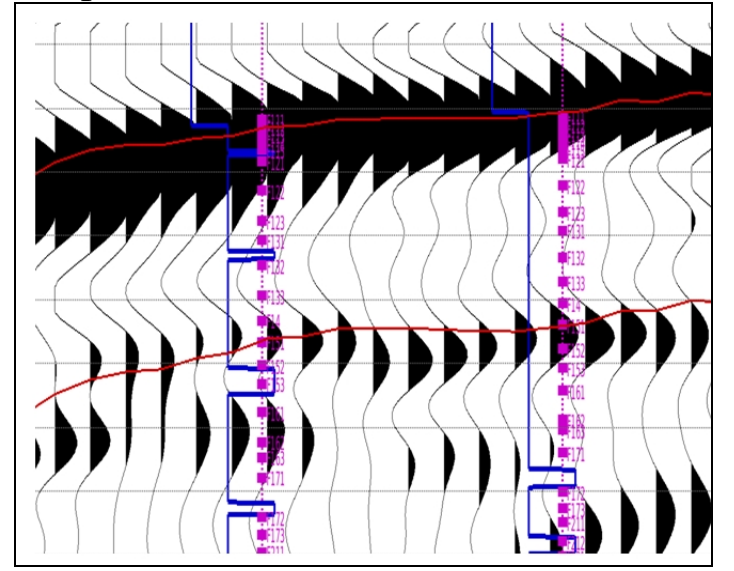

Fig.1. Seismic reflection characteristics comparison with lithology

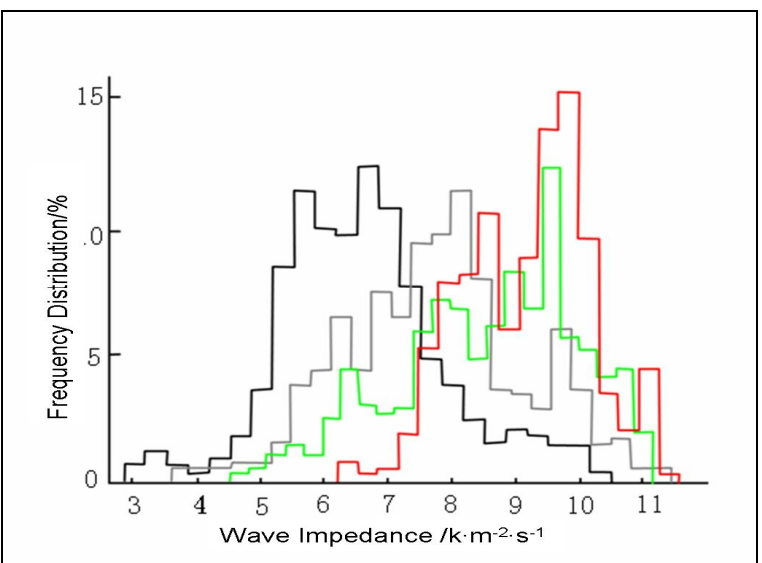

Fig. 2. Histogram of lithology and wave impedance

(red :sand,green:muddy siltstone,gray:Silty mudstone black: mudstone)

\section{Analysis}

Based on seismic data analysis (Fig.1), the seismic data in some areas of strong energy reflection characteristics is not correspondent with sand and mudstone lithology (Fig.1 the right well data). Therefore, it is necessary to analyze the real well data and forward simulation results to identify the solutions. 
Acoustic logging curve and impedance curve are the bridge between seismic and well logging. Through the analysis of the logging impedance wave and the logging interpretation result, the correlation between the wave impedance curve and the interpretation result is poor, and the identification of sand and mudstone can not be effectively carried out by using the wave impedance curve. Analysis of the cored wells results analysis found that there are a lot of transitional rocks (silty mudstone, muddy silty sandstone). Through the histogram analysis of lithology and wave impedance (Fig.2), the distribution of sand and mudstone is added by the transitional rocks, which makes the wave impedance curve can not be used for lithology identification. So the result of reservoir prediction using seismic data is not effective.

Comparing the synthetic seismogram from lithology wave impedance and logging wave (Fig.3), we found there exist wave groups from the influence of transitional rocks in seismic data and logging wave impedance synthetic seismic records. The seismic data cannot reflect the situation of the underground reservoir. By generating the difference of the forward modeling of the lithology wave impedance and the impedance curve of the well logging, the influence of transitional rocks on the actual seismic data can be removed.

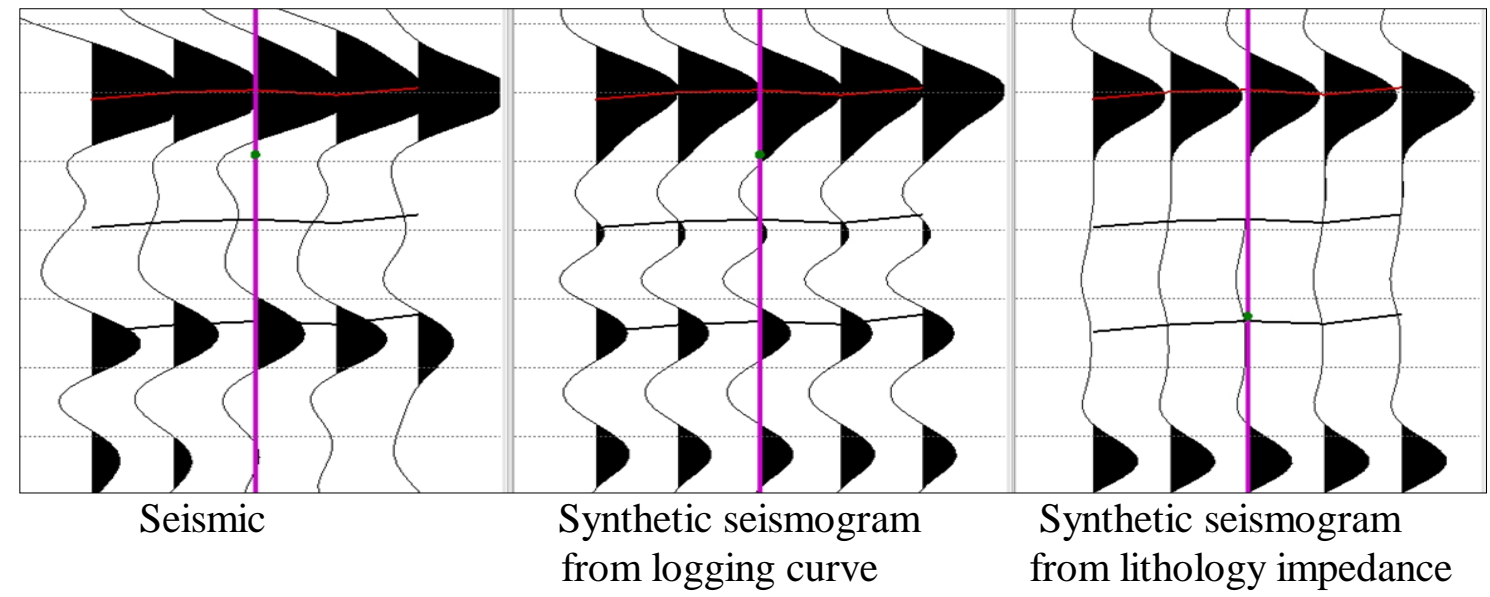

Fig.3. Synthetic seismogram from lithology wave impedance and logging wave

\section{Method}

The specific process is to use the "two steps" to achieve (Fig. 4). Based on the log wave impedance curve and lithology impedance curve, it is used to remove the influence of transitional rocks in the seismic data. Through the analysis of the relationship between lithologic assemblage characteristics and seismic horizon, the seismic properties are extracted from different time windows, and the purpose of reservoir prediction is achieved.

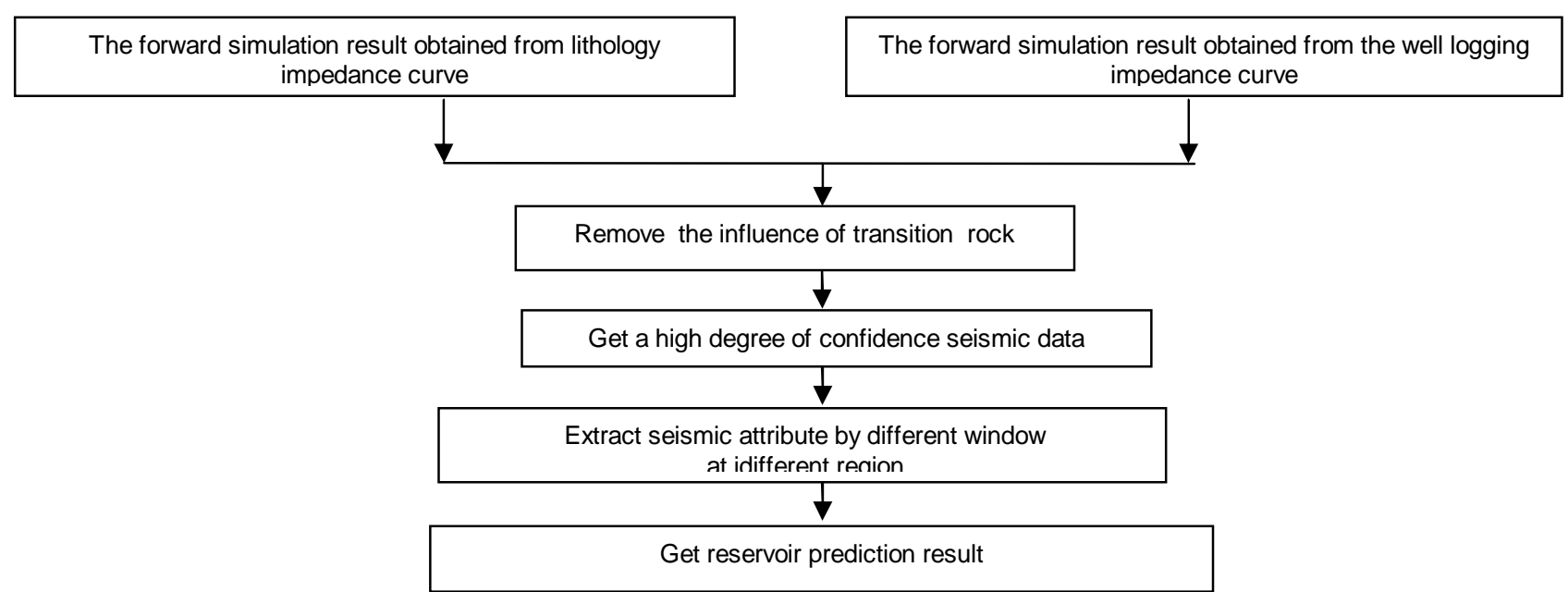

Fig.4.Research process 
The theory of forward modeling is mainly based on convolution method, ray tracing method and wave equation method. Convolution forward modeling method is relatively simple, that is, establish the geological model, calculate the reflection coefficient, and with the wavelet convolution calculation, so as to get the forward modeling seismic record. It has the characteristics of high speed, high efficiency, no need migration processing, and the result is less affected by the interference ${ }^{[2]}$.

The most important influence factor of convolution forward modeling is wavelet, which determines the shape of seismic data (including amplitude, phase, frequency). Wavelet in the space is both relatively stable, but also changes, affected by the explosive energization condition, the geological characteristics, and so on. In the past, the wavelet is fixed in the process of forward modeling, which makes the difference between seismic data and the forward modeling data. By using the spatial varied wavelets of forward modeling (Fig. 5), we can make the forward simulation and seismic data closer and the residuals smaller ${ }^{[3]}$. Wavelet body can extract using single well logging data and interpolate spatially.

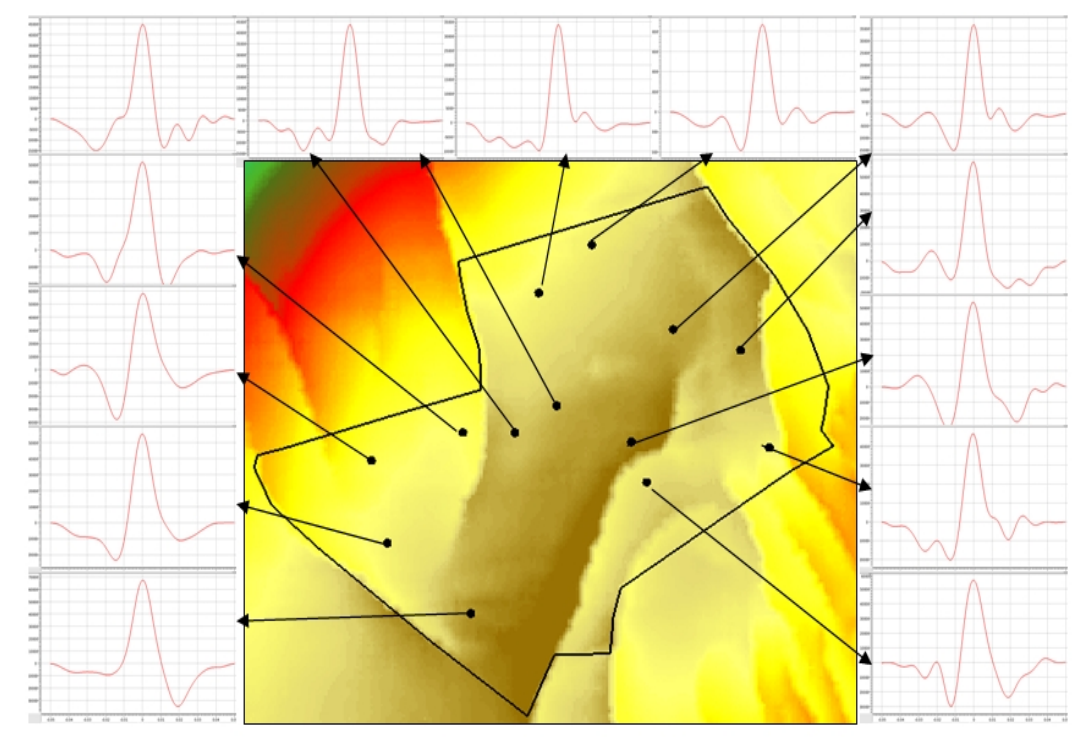

Fig.5. Spatial varied wavelets

The forward simulation result a obtained by convolution operation with wavelet body and the reflection coefficient from lithology impedance curve, only reflect the formation of mudstone and sandstone. The forward simulation result a removed transitional rocks (silty mudstone and mud silty sandstone). The forward simulation result $\mathrm{B}$ is used the reflection coefficient from the well logging wave impedance not lithology impedance curve, which is more close to the actual seismic data. Through the analysis of result A and B, when they are both positive or negative wave group the regional seismic data with high degree of confidence, and when the results appear in the axial direction is inconsistent with that seismic data can not be trusted, should be shielded. Through the judgment principles (Table 1), we can identify the region and range of the influence of the transition rocks, and then it can be shielded from the seismic data to obtain a high degree of confidence.

Table 1 judgment principles

\begin{tabular}{|c|c|c|}
\hline Result A & Result B & result \\
\hline Positive & Positive & Trusted (reserved) \\
\hline Positive & Positive & Untrusted(removed) \\
\hline Negative & Positive & Untrusted(removed) \\
\hline Positive & Positive & Trusted (reserved) \\
\hline
\end{tabular}

Through the analysis of different position result of well logging, seismic data and horizon, There are many styles in the relationship and the relative position to seismic marker. The seismic response 
characteristics are connected with lithology combination structure type. Due to the different rock combination can form a combination of different seismic reflection, we can extract seismic attribute along layer by different window size to carry out reservoir prediction. The selection of different regions of the window should be changed ${ }^{[4]}$.

Analyzing the relationship of the seismic horizon and seismic wave group characteristics at different region, we can determine the time window to extract seismic attribute of the high degree of confident seismic data. Then with the well thickness map and sedimentary facies diagram carries on the contrast analysis, we can find the better attribute slice results in different area and use the result of to compose stitching and synthesis reservoir prediction result (Fig.6).

The upper right region takes horizon down $3 \mathrm{~ms}$ in Figure 6, and on the left takes horizon down $5 \mathrm{~ms}$, right under the regional takes horizon down $1 \mathrm{~ms}$.

The final reservoir prediction results are compared with the sandstone thickness map, and the sedimentary facies map. The results are in line with the geological sedimentary law. Compared with the original reservoir prediction, the coincidence rate is increased from $53.9 \%$ to $75.1 \%$.

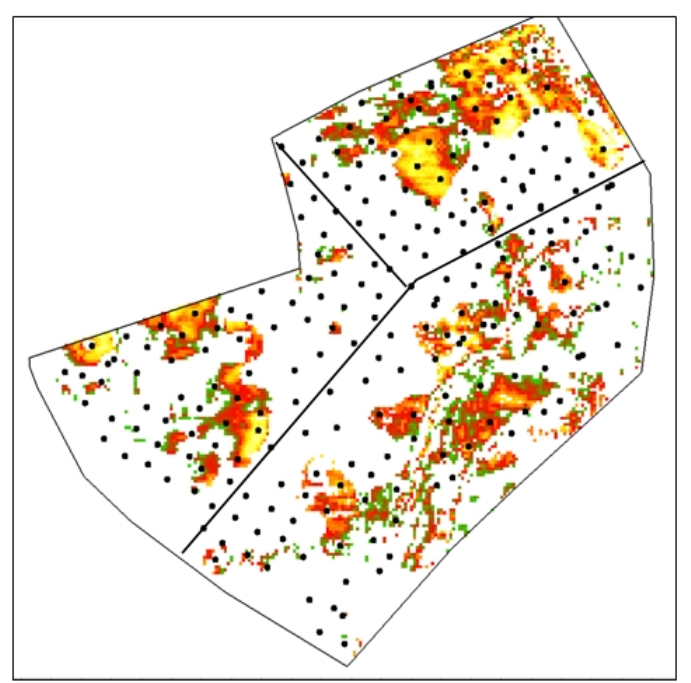

Fig.6. Synthesis reservoir prediction result

\section{Conclusions}

Using the logging interpretation, acoustic impedance log and wavelet body can generate different forward simulation results, and the forward difference between the results carry out the discrimination of the reliability of the seismic data, and get a highly reliability seismic data.

According to the different relationship and the relative position of the seismic response characteristics with seismic horizon, the determine seismic attributes in different regions is extracted with several windows, and carry out the reservoir prediction result. The results increase the accuracy of the reservoir prediction.

\section{Reference}

[1] LI Yanping, CHEN Shumin, SONG Yongzhong, et al . Petroleum Geology \& Oilfield Development in Daqing, Vol. 24(2005), p. 13-16

[2] Zhao Hu, YING Cheng, BAO Xiangsheng,et al . OGP, Vol.48 (2013), p. 728-733

[3]SUN Chengyu .Journal of the University of Petroleum, China. Vol.24 (2001), p. 77-84

[4]LIU Wei,YIN Cheng,WANG Min,et al.Geophysical prospecting for petroleum, Vol.53(2014), p. 468-476 